Classification

Physics Abstracts

$85.42+\mathrm{m}$

\title{
The nanometer age: Challenge and chance $\left({ }^{\star}\right)$
}

\author{
Heinrich Rohrer \\ IBM Research Division, Zurich Research Laboratory, CH-8803 Rüschlikon, Switzerland
}

(Received July 4; accepted October 27, 1994)

\begin{abstract}
The new players in the emerging nano-world are individual, selected objects of the size of some $50 \mathrm{~nm}$ down to molecules and atoms. The new aspect of science and technology on the nanometer scale is that these objects are treated as individuals, not as ensemble members. To a great extent, this requires real space methods. Local probe methods, such as scanning tunneling microscopy and its derivates, are therefore a key to the nano-world. Major challenges of the new nanometer world are to exploit the new possibilities that arise from nanometer dimensions, to interface the macroscopic world to nano-individuals, to establish new concepts for working with very large numbers of nanoindividuals and large sets of control parameters, to create the basis for broad interdisciplinarity, and to prepare society for the tremendous changes anticipated in a nanometer world.
\end{abstract}

\section{Introduction.}

Miniaturization is one of the key driving forces for science and technology on the nanometer scale. Figure 1 shows the progress of miniaturization for two examples from the data processing industry [1]. In the past two decades, miniaturization has progressed exponentially. The challenge in the coming decade, Period 1 in figure 1, will be to find methods suitable for the mass production of Gbit chips from those present-day elements that can already be miniaturized sufficiently and assembled in small quantities [3]. In Period 2, say, 10 to 20 years from now, the challenge will be to develop new types of elements. In both periods the investment into new technologies versus anticipated possible return will be a central problem. Eventually miniaturization, the division into ever smaller blocks, will come to an end in Period 3.

While solid-state science and technology have moved down from the millimeter to the nanometer scale, chemistry has simultaneously and independently progressed from the level of small, few-atom molecules to macromolecules of biological size (see Fig. 2). Supra-molecular chemistry might eventually provide the functional elements for the assembly scenario in the postminiaturization period. Biological elements in general might be impractical, but biological concepts will guide us to new ways of thinking and doing things. Numerical approaches have taken a

$\left.{ }^{\star}{ }^{\star}\right)$ Reprinted by permission of Kluwer Academic Publishers from Forces in Scanning Probe Methods H.J. Güntherodt, D. Anselmetti and E. Meyer, Eds., Proc. NATO ASI Conference, Schluchsee, Germany, March 7-18, 1994 (NATO ASI Series E: Applied Sciences, in press). 


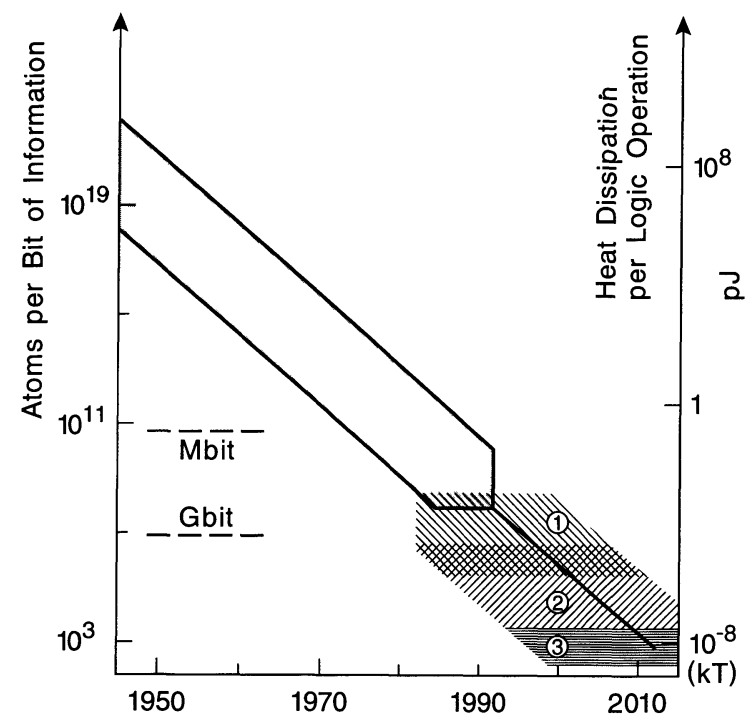

Fig. 1. - Progress of miniaturization in information technology. From reference [2], (C) Società Italiana di Fisica.

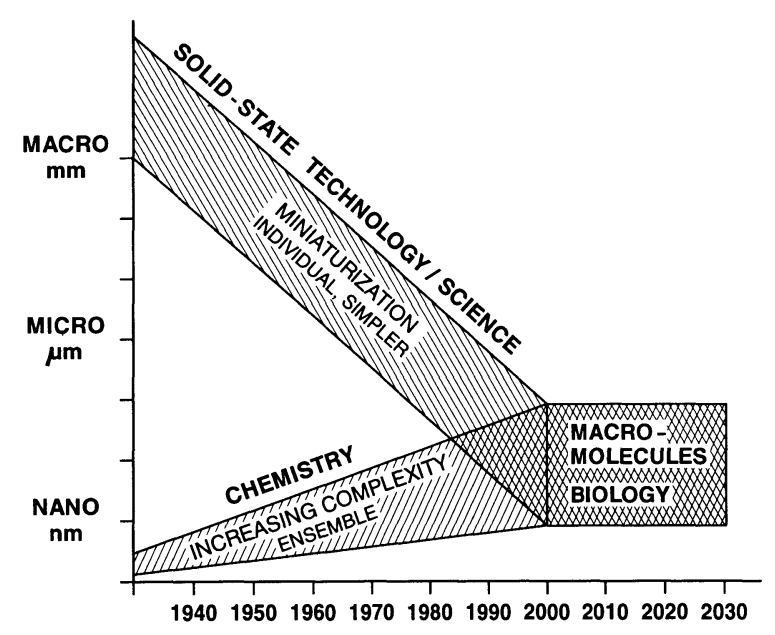

Fig. 2. - Developments in solid-state technology and chemistry: miniaturization builds on ever smaller individuals; increased complexity of ensemble members distinguishes macro-molecular chemistry. From reference [4].

similar development like that of chemistry, from atoms and small molecules to ever larger nanoobjects. They will be of great importance in understanding properties, functions and processes on the nanometer scale because on the one hand theory has little symmetry and no fixed dimensionality to build on and on the other hand functions and processes of nanometer-sized elements depend critically on their immediate environment. 


\section{The new nanometer world.}

The nanometer age can thus be considered as a continuation of an ongoing development: for example, miniaturization in solid-state technology, increasing complexity in chemistry and numerically intensive computation. However, the new possibilities and novel aspects when working with nanometer dimensions go far beyond that - beyond, e.g., "smaller, faster, cheaper" in information technology. Dealing with chemical bonds rather than bulk mechanical properties leads to a new nano-mechanics with, for example, strains an order of magnitude larger than those in the bulk [5]. Mechanical resonance frequencies in the $\mathrm{MHz}$ to $\mathrm{GHz}$ range [6, 7] and thermal and diffusion response times below nanoseconds should very well complement fast electronics. Local electric fields of up to several volts per angstrom, which are attainable in a scanning tunneling microscope (STM) configuration, and chemical interaction forces at angstrom distances are the basis for manipulation and modification on the atomic and the molecular level [8-11]. Forces on the nano-scale are therefore a key to the nano-world. In addition, the extremely high electric fields provide convenient access to local nonlinear phenomena [12] and might also revive applications of thin ferroelectrical films. Other interesting and challenging aspects of nano-scale dimensions comprise the following. Quantum effects will become important; we have to deal with tera and peta individuals; we often will think in terms of single electrons rather than currents; the immediate environment is a vital part of the nano-individual and not just a linear, minor perturbation; parallel operation will become the norm, and assembly and self-organization will replace miniaturization procedures.

Progress after miniaturization will be based on increased complexity. A promising route could be the assembly of molecular-sized functional elements into complex functional units. A primary task of science is to find appropriate self-assembling techniques and ways to interface the macroscopic world with molecular-sized functional elements for communication and control or modification of their functions. This will lead to an extremely fruitful, interdisciplinary effort that is expected to add new dimensions to biology as well as to supramolecular chemistry. The coming nanometer age can, therefore, also be called the age of interdisciplinarity.

Major tools for the nanometer world comprise beam methods (microscopy and lithography with beams of electrons, ions, photons, atoms, maybe sometime even with neutrons and positrons), local probe methods (STM and its derivates), computational methods and new nano-materials. The beam methods are the chief fabrication methods of current microtechnology, which they will carry deep into the nanometer age. In the nano-world, these methods might be the key for producing the patterns necessary for self-assembly and self-organization of and for communication with nanoindividuals. The computational methods will be central for theory, as mentioned above, both in terms of understanding properties and processes on the nanometer scale as well as in context with new concepts of handling very large numbers of nano-individuals and many degrees of freedom in systems of nano-individuals. New nano-materials are required for machining on the nanometer scale as well as for providing appropriate supports of nano-objects, be they particular biological molecules, macro molecules from supramolecular chemistry or building blocks from solid-state technology.

\section{Local probe methods.}

Local probes are the "finger tips" to interact with nano-individuals, very much in the same way as we sense and handle macroscopic materials with our fingers. The positions and the properties of objects and functions as well as of processes associated with them are sensed, conditioned or changed by interactions between the probe and the object. 
The distance dependence of the interaction is the key to the sample topography. Scanning at constant interaction gives a constant-interaction contour that reflects the sample topography, provide the interaction is laterally homogeneous. However, the ability of gaining access to inhomogeneities down to the atomic scale is one of the unique and attractive features of the local approach and of atomic-scale imaging. The more inhomogeneous the objects of interest are, i.e., for the truly "colorful" and interesting objects, the more important it becomes that the probeobject distance can be controlled independently of the experiment to be performed. Also, a local probe measurement usually includes different interactions. In tunneling, for example, the interactions are the overlaps of tip and sample electronic wave functions at equal energy - inelastic processes are smaller by several orders of magnitude - thus different electronic states with different wave-function overlaps contribute to the total tunnel current. The art of local probe methods is then to find one interaction to control the probe-object distance and one to perform the experiment, and to separate either interaction from all the others, i.e., separation into a control and a working interaction, respectively. Ideally, the control interaction should be monotonous and, for imaging, laterally homogeneous. It is, therefore, the appropriate interaction for imaging the topography.

For most of the classical surface-science-type STM experiments, the interaction separation can be handled to a great extent by tunneling spectroscopy. The preparation methods yield compositionally well-defined surfaces of long-range homogeneity. Short-range inhomogeneities are periodic or easily recognizable, such as steps and defects - yet by no means does this imply "easy" experiments. In most other cases, however, interaction separation is essential for understanding images. In general, separation requires simultaneous measurement of two or more quantities. In magnetic force imaging, for example, the separation of the magnetic forces and their lateral variation from the other forces and topographic effects can be achieved by introducing a well-defined Coulomb force [13]. For ambient imaging, a procedure to separate the topography from electronic and elastic effects has recently been proposed that requires the simultaneous measuring of force and compliance on a constant tunnel current contour [14]. Artifacts can arise when the various interactions involved yield different image resolution. Then the interaction with least resolution - or the least inhomogeneous interaction from the imaging point of view — is the most suitable one to be used as control interaction.

It should be noted that "topography" is not a clear-cut thing. Topography as the smoothed average position of surface atoms, although probe-independent, is of limited practical value for surfaces with different atoms of different sizes. A best compromise for the topography and thus also for the probe-object distance might be the point of zero force or point of contact between object and probe apex atom, although this topography can be tip dependent. The corresponding control interaction is then the total force between object and probe apex atom. Unfortunately this force is not accessible in a force measurement, which yields only forces between the object and the entire probe. Working in a liquid eliminates [15] or substantially reduces some of the less local forces, however, molecules squeezed between probe and sample can complicate matters. Nevertheless, determining the point of contact by, for example, an abrupt change in damping or effective lever compliance [16] appears at present to be a truly meaningful way to define within some thenths of an angstrom and control the probe-object distance.

Subsurface sensitivity is achieved when the interaction extends into the object, e.g., the electrostatic interaction of a conducting or polarizable probe with an electronic charge in an insulating layer [17]. This, however, results in a loss of resolution, since physically the probe cannot come closer than the object surface. Other subsurface methods include ballistic electron emission microscopy (BEEM) [18], in which ballistic electrons injected by a tunnel tip probe electronic properties at buried interfaces, and local luminescence [19] of quantum-well structures, where the emitted light from the recombination of injected electrons is characteristic of both the surface 


\section{Measurement:}

(a)

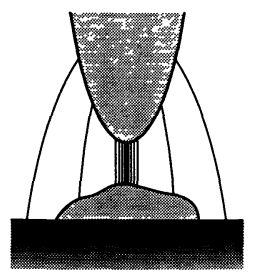

Tunneling (b)

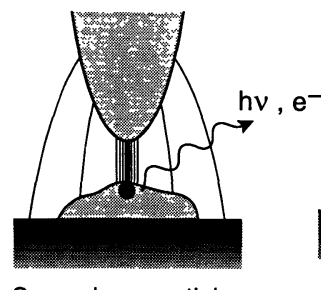

Secondary particles (c)

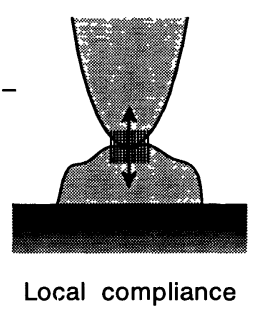

Probe-Object Configuration:

(d)

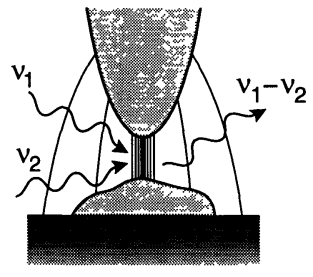

Interaction with light

Tool:

(g)

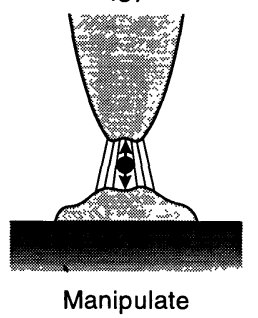

(e)

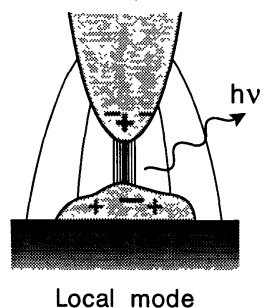

(h)

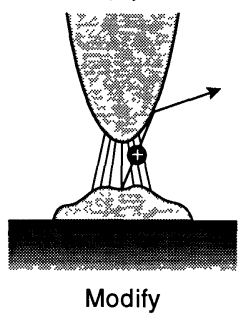

(f)

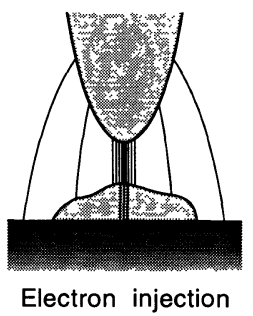

(i)

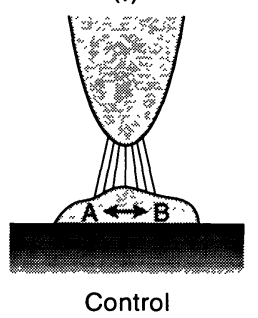

Fig. 3. - Local probe methods for imaging (a-c), as part of a specific experimental configuration on the nanometer scale (d-f), and as tool (g-i). From reference [20].

band bending and the band gap in the interior.

A first set of applications of local probe methods deals with measurement, i.e., to monitor displacements, to determine when contact occurs, to measure local properties and to perform imaging (see Figs. 3a-c). The interactions should of course not affect the properties under consideration, although they might change others. A second set uses special aspects of the probeobject configuration. In figure $3 \mathrm{~d}$, the nonlinearity of the tunnel junction mixes different light frequencies. This can be used to image a property via the nonlinearity of the junction $[12,21]$ or to use a particularly strong local nonlinearity for frequency mixing per se [22]. In figure $3 \mathrm{e}$, a local plasmon mode characteristic of the tip-sample system is excited by the tunneling electron, which on decay emits a photon [23]. In figure 3f, the injected electrons are used to probe a buried interface by BEEM [18] or to investigate surface and bulk semiconductor band edges, for example, in quantum-well structures [19]. Finally, the local probe can serve as manipulator or as machining tool. Rearranging adsorbed atoms and molecules on surfaces has resulted in most remarkable structures such as atom corrals [11]. In figure $3 \mathrm{~g}$ an atom is switched back and forth between tip 
and surface - the atom switch [24]. Extraction (Fig. 3d) and deposition of clusters and even of individual atoms have opened an exciting area of surface modification [8-11]. Finally, control of processes and functions (Fig. 3i) is one of the ultimate aims of science and technology on the nanometer scale.

\section{Competence and challenges.}

We have already acquired considerable competence with simple model systems. Atomic resolution imaging of structural, electronic and mechanical properties and of the growth and diffusion phenomena under various conditions, ranging from ultra-high vacuum to electrochemical environments, has become standard. For less simple systems, however, separation and individual control of the interactions involved in a local experiment is crucial for understanding of the imaging process and interpretation of the image. Images can be beautiful and interesting, but then so is a sphinx. Force measurements are expected to play a central role for further progress in local characterization methods. Further challenging issues concern magnetic properties and chemical specificity on a nanometer scale and the combination of very short time scales in the microsecond and picosecond range with nanometer-scale resolution [25]. Local probe methods have also brought a quantum leap for in situ characterization of electrochemical systems and have opened exciting prospects for surface modifications on the nanometer scale by electrochemical means [26].

The electrode-electrolyte interface is tremendously rich, with all its reconstructions and other structural and compositional phenomena of no lesser variety than those of the solid-vacuum interface in classical surface science. Moreover, the composition of the electrolyte brings and additional degree of freedom, reflected for example in the electrolyte-dependent reconstructions [27].

Nano-electrochemistry has taught us that local probe methods can be applied equally well in liquid environments. Therefore, it has pioneered the nanoscopic approach to the solid-liquid interface in general. The central importance of understanding and controlling the solid-liquid interface on a nanoscopic scale, however, extends far beyond the classical topics of electrochemistry [20].

Liquids provide new ways to treat and control surfaces. Capillary and van der Waal's forces acting on cantilever force sensors in force microscopy are best controlled in liquids [16]. We can also think of surface control through passivation with a liquid and simultaneous local surface modification using, for example, specific molecules in the liquid [28].

A new surface science will emerge that can deal with "real" surfaces at ambient conditions and in liquids, and which is based on the extremely high resolution of local probe methods and their adaptability to different environments. This could open the present surface science of homogenized, well-prepared, well-controlled and reasonably well-defined surfaces to a large variety of "real" surfaces and interfaces that can be inhomogeneous on the smallest possible scale.

Important for such a new type of surface science, however, is a much improved chemical analysis capability of local probe methods. Characterization of "real" surfaces and interfaces will involve different types of experiments, since initially much less is known about the state of such surfaces and interfaces than about that of well-prepared and controlled surfaces. For interaction separation, the experiments have to be performed simultaneously, especially because "real" interfaces can neither be reproduced on a local scale nor sufficiently controlled for sequential local experiments. The local approach will also produce very large data sets for representative surface samples, calling for increased speed and parallel operation as well as for new ways of handling and analyzing such volumes of data.

Local probe methods give us the ability to interact with individual functional molecular units, be 
it to study or to control their functions and the processes associated with them. The functionality of most of them, such as those of biological molecules, depends critically on an appropriate liquid environment. Therefore, mastering the solid-liquid interface on a nano-scale is crucial to the application of local probe methods to in vivo biology.

Lastly, the liquid provides the third dimension for efficient self-assembly and self-organization of large molecules on surfaces. Such "selfprocedures" will play a central role in the emerging nano-age, where we will have to build and interact with tera and peta nanometer-sized objects on an individual or at least on a distinctly selective basis.

The liquid-solid interface, quite generally, is a crucial element for interfacing the macroscopic world to nano-individuals - one of the primary objectives and challenges of science and technology on the nanometer scale.

Manipulation and modification on the nanometer or even atomic scale have made tremendous progress in the past couple of years [8-11]. Some view these efforts mainly as a road leading to large-scale integrated systems, e.g., petabyte memories. Whether simple scratching with storage densities of Gbits $/ \mathrm{cm}^{2}$ [29], atom extraction or deposition [9] at $100 \mathrm{Tbits} / \mathrm{cm}^{2}$ or other methods with performances somewhere in between will ever lead to viable large-scale storage application is an open issue. This will depend crucially on the possibility of producing miniaturized nanotools suitable for parallel operation, for example of thousands to millions of tips as reading and writing heads, as well as on the progress in current technologies. However, even more exciting might be the prospects of creating sophisticated and complex nano-structures and nano-machines by manipulation and modification. Such nano-machines would be used for specific experiments or could perform specific tasks that cannot be reasonably executed or are even impossible by other means. The simplest nano-machine - although it is far from being simple - could be a functionalized tip with a specific test molecule attached that is used for recognition of other molecules [20,30]. Mbit to Gbit memories of micro- to millimeter dimension, everything included, could have many applications for "local" tasks. Finally, the multibillion-dollar human genome project could essentially be miniaturized, in a first step, to a local-probe DNA imaging station and in a second step to a biological DNA reading unit with an appropriate interface to the human world. We have again "smaller, faster, cheaper", but applied to complex tasks not to individual elements. For instance, the cost of memory bits in a nano-machine plays a lesser role than for mass storage. Local probe methods appear indispensable in the exploratory stage of the nano-world. Once standard, however, fabrication of nano-machines and their control might be achieved by other means.

Miniaturized sensors and actuators requiring nano- to picometer precision and control are another rewarding challenge. They will serve as local measuring and control stations and as sensory organs, hands and feet of nano-robots, i.e., small robots working with nm-to-pm precison. An example is the micro-calorimeter [31], which measures pJoules of reaction heat in msec, and we can readily envisage the ability to measure fJoules in $\mu$ sec.

Quite generally, the nanometer will become the new standard of precision. Micrometer precision was a crucial element for the later part of industrialization and for the beginning of the technology age. Although already accepted as the new standard for microtechnology of the near future, the notion of a nanometer world still encounters considerable reservation in the western industrial world at large. To change that is indeed a challenge. It might help to remember that the micrometer had no significance for a farmer plowing his field with an ox and plow 150 years ago - not for the ox or the plow. Nevertheless, the micrometer changed plowing - it is the precision standard for the tractor.

Interfacing the macro-world with nano-individuals is one of the great challenges. Figure 4 sketches a program for the case of functional biological macromolecules. In the first step shown in figure $4 \mathrm{a}$, neither the substrate nor the probe are activated; the molecule is physisorbed directly 
(a)

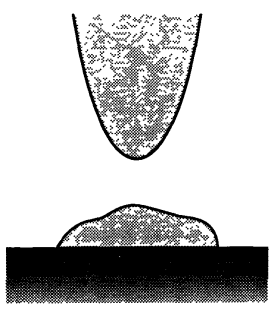

(c)

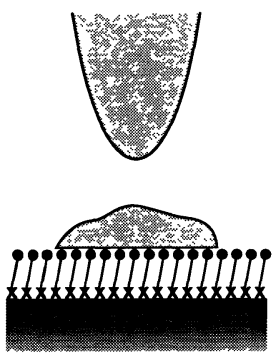

(e)

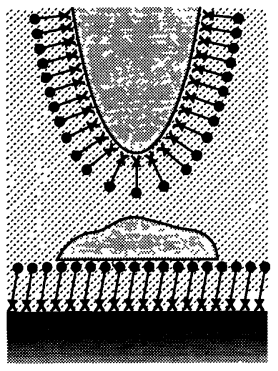

(b)

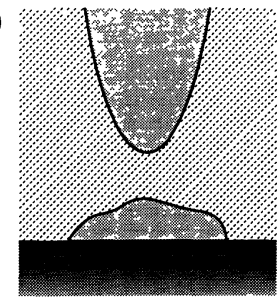

(d)

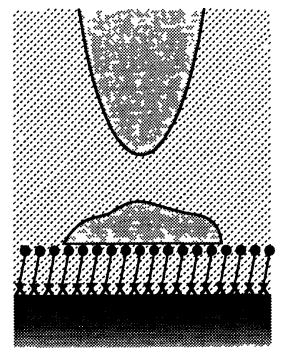

(f)

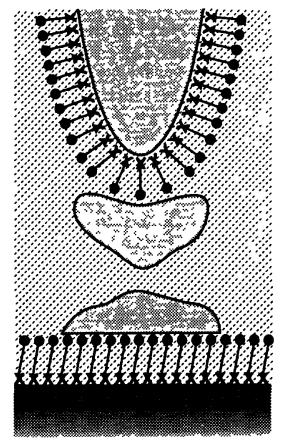

Fig. 4. - Program for "interfacing molecules" by chemical activation (functionalizing) of probe and substrate. a) "Bare" configuration, b) "functional" environment, c) activation of substrate, here by a selfassembled monolayer, d) configuration c) in proper environment, e) configuration d) with activated probe. Steps a) to e) connect a functional object via the functional probe with the outside world. In $\mathrm{f}$ ) a functional molecule is the new probe. From reference [20].

onto the substrate [32]. This step is used for qualitative imaging and for exploring communication with the molecule. In figure $4 \mathrm{~b}$, object and probe are immersed into an appropriate liquid environment. Of interest here is the immobilization in a liquid environment for imaging the "true" shape of the molecule and for communication. In figure $4 \mathrm{c}$, the molecule is immobilized on a self-assembled monolayer - a problem currently of interest [33]. The next steps include immobilization in the proper environment on a chemically activated substrate (Fig. 4d) and finally activation of the probe (Figs. $4 \mathrm{e}$ and $4 \mathrm{f}$ ).

Progress into the nanometer age depends critically on improved interdisciplinary thinking and acting, both within science and between science and engineering. The thinking starts in the heads of scientists and in those of open-minded money agencies, the acting begins in formulating interdisciplinary projects and subsequent cooperation between scientists who are well trained in their 
disciplines. Interdisciplinarity is manily a matter of the attitude of the scientific community - not of science politics.

Being able to handle condensed matter on an atom-by-atom basis opens tremendous perspectives, but also fears. Both engender the wish for controlling science. The destiny of society, however, lies in the proper use of science, not in its control.

\section{References}

[1] Keyes R.W., IBM J. Res. Develop. 32 (1988) 24.

[2] Rohrer H., Proc. EPS 9 Conference "Trends in Physics", Florence, Italy, Sept. 1993, in: II Nuovo Cimento, Ser. 2, 107A, No. 7 (1994) 989.

[3] Wind S.J., Reeves C.M., Bucchignano J.J., Lii Y.T., Newman T.H., Klaus D.P., Keller J., Volant R.P., Trebin B. and Hohn F.J., J. Vac. Sci. Technol. B10 (1992) 2912, 2917.

[4] Rohrer H., Ultramicroscopy $42-44$ (1992) 1.

[5] Pethica J., in Forces in Scanning Probe Methods, Proc. NATO Adv. Study Institute, Schluchsee, Germany, March 7-18 (1994), NATO ASI Series E: Applied Sciences (Kluwer, Dordrecht, to appear), and in reference [8d].

[6] Rugar D., Yannoni C.S. and Sidles J.A., Nature 360 (1992) 563; Rugar D., Züger O., Hoen S., Yannoni C.S., Vieth H.-M. and Kendrick R.D., Science 264 (1994) 1560-1563.

[7] Vu Thien Binh, Garcia N. and Levanyuk A.L., Rev. Sci. Instrum. 63 (1992) 1124 and Surf. Sci. Lett. 301 (1994) L224.

[8] For reviews see (a) C.F. Quate, in: Highlights in Condensed Matter Physics and Future Prospects, L. Esaki Ed., NATO ASI Series B, 285, 573 (Plenum, New York, 1991); (b) Nanosources and Manipulation of Atoms under High Fields and Temperatures: Applications, Vu Thien Binh, N. Garcia and K. Dransfeld Eds., NATO ASI Series E, 235 (Kluwer Academic, Dordrecht, 1993); (c) Atomic and Nanometer-Scale Modifications of Materials: Fundamentals and Applications, Ph. Avouris Ed., NATO ASI Series E, 239 (Kluwer Academic, Dordrecht, 1993); (d) Proc. NATO ARW The Ultimate Limits of Fabrication and Measurement, Cambridge, England, April 5-8, 1994 (to appear).

[9] García R., Appl. Phys. Lett. 60 (1992) 1960; Hosoki S., Hosaka S. and Hasegawa T., Appl. Surf. Sci. 60/61 (1992) 643; Aono M., Kobayashi A., Grey F., Uchida H. and Huang D.H., Jpn. J. Appl. Phys. 32 (1993) 1470; Euchs H., Schimmel Th., Akari S., Eng L.M., Anders M., Lux-Steiner M. and Dransfeld K., in reference [8b], 293.

[10] Lyo I.-W. and Avouris Ph., Science 253 (1991) 173 and reference [8c], 11.

[11] Eigler D.M. and Schweizer E.K., Nature 344 (1990) 524; Crommie M.F., Lutz C.P. and Eigler D.M., Science 262 (1993) 218.

[12] Kochanski G.P., Phys. Rev. Lett. 62 (1989) 62; Michel B., Mizutani W., Schierle R., Jarosch A., Knop W., Benedickter H., Bächtold W. and Rohrer H., Rev. Sci. Instrum. 63 (1992) 4080; Mizutani W., Michel B., Schierle R., Wolf H. and Rohrer H., Appl. Phys. Lett. 63 (1993) 147.

[13] Schönenberger C. and Alvarado S.F., Z. Phys B80 (1990) 373.

[14] Anselmetti D., Gerber Ch., Michel B., Güntherodt H.-J. and Rohrer H., Europhys. Lett. 23 (1993) 421.

[15] Marti O., Drake B. and Hansma P.K., Appl. Phys. Lett. 51 (1987) 484; Goodman F.O. and Garcia N., Phys. Rev. B43 (1991) 4728; Garcia N. and Vu Thien Binh, Phys. Rev. B46 (1992) 7946; Ohnesorge F. and Binnig G., Science 260 (1993) 1451.

[16] Bining G., US Patent 4724 318; Delain E., Fourcade A., Poulin J.-C., Barbin A., Coulaud D., Cam E. and Paris E., Microsc. Microanal. Microstruct. 3 (1992) 457; Elings V. and Gurley J., US Patent 5266 801.

[17] Schönenberger Ch. and Alvarado S.F., Phys. rev. Lett. 65 (1990) 3162.

[18] Kaiser W.J. and Bell L.D., Phys. Rev. Lett. 60 (1988) 1406; Ludeke R., Phys. Rev. Lett. 70 (1993) 214; Ludeke R. and Bauer A., ibid 71 (1993) 1760; Niedermann Ph., Quattropani L., Solt K., MaggioAprile I. and Fisher O., Phys. Rev. B48 (1993) 8833; Sirringhaus H., Lee E.Y. and von Känel H., Phys. Rev. Lett. 73 (1994) 577, ibid. Surf. Sci. 314 (1994) L823.

[19] Alvarado S.F. and Renaud P., Phys. Rev. Lett. 68 (1992) 1387; Vasquez de Parga A.L. and Alvarado S.F., Phys. Rev. Lett. 72 (1994) 3726. 
[20] Rohrer H., Surf. Sci. 299/300 (1994) 956.

[21] Krieger W., Koppermann H., Suzuki T. and Walther H., IEEE Trans. Instrum. Meas. (U.S.A.) 38 (1989) 1019; Krieger W., Susuki T., Völcker M. and Walther H., Phys. Rev. B41 (1990) 10229; Völcker M., Krieger W. and Walther H., J. Vac. Sci. Technol. B 12 (1994) 2129.

[22] Sullivan T.E., Kuk Y. and Cutler P., IEEE Trans. Electron Devices 36 (1989) 2659; Nyguen H.Q., Cutler P.H., Feuchtwang T.E., Huang Z., Kuk Y., Silverman P.J., Lucas A.A. and Sullivan T.E., ibid 36 (1989) 2671; kuk Y., Becker R.S., Silverman P.J. and Kochanski G.P., Phys. Rev. Lett. 65 (1990) 456.

[23] Berndt R., Gimzewski J.K. and Johansson P., Phys. Rev. Lett. 67 (1991) 3796; Berndt R., Gaisch R., Schneider W.D., Gimzewski J.K., Reihl B., Schlittler R.R. and Tschudy M., Appl. Phys. A57 (1993) 513.

[24] Eigler D.M., Lutz C.P. and Rudge W.E., Nature 352 (1991) 600.

[25] Yokoyama T. and Inoue T., Thin Solid Films (in press); Inoue T., Jeffery M.J. and Yokohama H., in Forces in Scanning Probe Methods, Proc. NATO ASI Schluchsee, Germany, March 7-18 (1994), NATO ASI Series E: Applied Sciences (Kluwer, Dordrecht) (to appear); Hou A.S., Ho F. and Bloom D.M., Electron. lett. 28 (1992) 2305.

[26] Nanoscale Probes of the Solid Liquid Interface, A.A. Gewirth and H. Siegenthaler Eds., NATO ASI Series (Kluwer Academic, Dordrecht, to appear 1994).

[27] Chun-hsien Chen and Gewirth A.A., Ultramicroscopy 42-44 (1992) 437; Magnussen O.M., PhD Thesis, University of Ulm, Germany (1993); idem, in: Frontiers in Electrochemistry, J. Lipkowski and P.N. Ross Eds., 2 (VCH, New York, 1993).

[28] Foster J., Frommer J. and Arnett P., Nature 331 (1988) 324.

[29] Jung T.A., Moser A., Hug H.J., Brodbeck D., Hofer R., Hidber H.R. and Schwarz U.D., Ultramicroscopy $42-44$ (1992) 1446.

[30] Lee G.U., Kidwell D.A. and Colton R.J., Langmuir 10 (1994) 354.

[31] Gimzewski J.K., Gerber Ch., Meyer E. and Schlittler R.R., Chem. Phys. Lett. 217 (1994) 589.

[32] Bining G. and Rohrer H., in: Trends in Physics 1984, J. Janta and J. Pantoflicek Eds., 1 (European Physical Society, Prague, Czech Republic, 1985) 38.

[33] Häussling L., Michel B., Ringsdorf H. and Rohrer H., Angew, Chem. Int'l Ed. engl. 30 (1991) 569; B. Michel, Presentation at 2nd CEC Workshop on Bioelectronics "Interfacing Biology with Electronics" (Frankfurt/Main, Germany, Nov. 23-26, 1993). 